\title{
Rotavirus and adenovirus infections in children during COVID-19 outbreak in Hangzhou, China
}

\author{
Wei Li ${ }^{1}$, Yueling Zhu' ${ }^{2}$ Jingan Lou ${ }^{3}$ Jie Chen ${ }^{3}$, Xiaojun Xie ${ }^{1}$, Jianhua Mao ${ }^{4}$ \\ ${ }^{1}$ Department of Clinical Laboratory, The Children's Hospital, Zhejiang University School of Medicine, National Clinical Research Center for Child \\ Health, National Children's Regional Medical Center, Hangzhou, China; ${ }^{2}$ Department of Traditional Chinese Medicine, The Children's Hospital, \\ Zhejiang University School of Medicine, National Clinical Research Center for Child Health, National Children's Regional Medical Center, \\ Hangzhou, China; ${ }^{3}$ Department of Gastroenterology, The Children's Hospital, Zhejiang University School of Medicine, National Clinical Research \\ Center for Child Health, National Children's Regional Medical Center, Hangzhou, China; ${ }^{4}$ Department of Nephrology, The Children's Hospital, \\ Zhejiang University School of Medicine, National Clinical Research Center for Child Health, National Children's Regional Medical Center, \\ Hangzhou, China \\ Contributions: (I) Conception and design: W Li, J Mao; (II) Administrative support: J Lou, J Chen; (III) Provision of study materials or patients: W Li, \\ Y Zhu; (IV) Collection and assembly of data: W Li, X Xie; (V) Data analysis and interpretation: W Li, Y Zhu; (VI) Manuscript writing: All authors; \\ (VII) Final approval of manuscript: All authors. \\ Correspondence to: Jianhua Mao. The Children's Hospital of Zhejiang University School of Medicine, National Clinical Research Center for Child \\ Health, National Children's Regional Medical Center, 3333 Binsheng Road, 310052 Hangzhou, China. Email: maojh88@zju.edu.cn.
}

Background: This study aims to investigate the impact of protective measures and isolation on intestinal infection in children before and after COVID-19 outbreak in Hangzhou, China.

Methods: Data on outpatient visits, intestinal infection visits, and tests of adenovirus or rotavirus from electronic healthcare records were extracted in Children's Hospital of Zhejiang University School of Medicine during the COVID-19 outbreak (January-December, 2020), and be compared with those in 2019 during the same period.

Results: We found that pediatric outpatient visits, patients with intestinal infection and its proportion from January to December of 2020 were significantly less than that in the same period of 2019. Furthermore, the numbers of outpatient visits and patients with intestinal infection were the lowest in February and elevated slowly from March to December in 2020. We also found significantly decreased number of completed tests for intestinal viruses, positive cases of adenovirus and rotavirus, positive rate of rotavirus during JanuaryDecember, 2020. The positive rates of adenovirus from March to September and December of 2020 were significantly lower than the levels of the same period in 2019. The positive rate of rotavirus and adenovirus in all age groups in 2020 is lower than the level of all age groups in 2019.

Conclusions: The outpatient visits and intestinal, rotavirus and adenovirus infections in children significantly decreased during COVID-19 outbreak. Adequate protective measures and isolation in children may help to prevent intestinal virus infections in children.

Keywords: Rotavirus; adenovirus; COVID-19; children

Submitted Apr 02, 2021. Accepted for publication Jun 29, 2021.

doi: $10.21037 / \mathrm{tp}-21-150$

View this article at: https://dx.doi.org/10.21037/tp-21-150

\section{Introduction}

Acute diarrhea is one of the most common illnesses worldwide, and has a great impact on children (1). Among multitudinous pathogenic factors, rotaviruses and adenovirus are the most important viral causes of acute gastroenteritis in children (2). Both rotaviruses and adenovirus are mainly transmitted by fecal-oral transmission. Rotavirus infections can lead to severe dehydration, electrolyte disorders and 
acid-base disturbances (3). Adenoviruses are also major pathogens causing gastroenteritis, which can be transmitted throughout the year (4).

After the outbreak of the novel coronavirus disease (COVID-19), the Chinese government issued a series of strict and active prevention and control measures such as restriction of travel, social distancing, home quarantine, and wearing of masks since January 23, 2020 (5). Due to these active strategies, COVID-19 has been successfully contained in China. During the epidemic, not only societal behavior but also individual behaviors changed. As for children, most of them were asked to stay at home, routinely wear masks and frequently wash hands (6).

In addition to COVID-19, these prevention and control measures may also block the transmission routes of other viral infections. This retrospective study was conducted to investigate the influence of social isolation and the changes of human behavior and habits on intestinal infections, with a focus on rotavirus and adenovirus in children during the COVID-19 outbreak from January-December, 2020. The corresponding data in the same months in 2019 were utilized for comparison.

We present the following article in accordance with the MDAR reporting checklist (available at https://dx.doi. org/10.21037/tp-21-150).

\section{Methods}

The study was conducted in accordance with the Declaration of Helsinki (as revised in 2013). The study was approved by the ethics committee of the Children's Hospital of Zhejiang University School of Medicine (2020-IRB-016) and informed consent was taken from all the patients.

\section{Patients}

Data on outpatient visits and intestinal infections, number of completed tests for rotavirus and adenovirus antigen assays, and the confirmed positive cases from January-December, 2020 were collected from the electronic healthcare records at the Children's Hospital, Zhejiang University School of Medicine. The data were compared with those acquired in 2019 during the same period. Intestinal infections included primary diagnosis of enteritis, diarrhea, indigestion, gastroenteritis, and vomiting.

\section{Antigen test for detection of rotavirus $A$ and buman adenovirus}

Antigen test kit (Abon Biopharm Co., Ltd.) (7), approved by the Food and Drug Administration (FDA) of China (Approval No. 20153402309), was used for the detection of viral infections. About $50 \mathrm{mg}$ of stool samples were mixed with $1 \mathrm{~mL}$ of sample extraction reagent. The well plate was composed of four major parts: (I) a sample well plate; (II) a control line; (III) a rotavirus test line; and (IV) an adenovirus test line. Two drops (about $80 \mu \mathrm{L}$ ) of the sample mixture were added into a sample well plate. After 10-20 minutes of incubation, the sample was determined as rotavirus positive if both the control line and the rotavirus test line turned blue. If the control line was blue and the adenovirus test line was red, the sample was determined as adenovirus positive.

\section{Statistical analysis}

The results were analyzed using SPSS software (version 20.0). $\chi^{2}$ test was used to determine statistical differences. Two-tailed $\mathrm{P}$ values $<0.05$ were considered to be statistically significant.

\section{Results}

Firstly, we compared the numbers of outpatient visits from January to December between 2019 and 2020 at the Children's Hospital of Zhejiang University School of Medicine. As shown in Figure $1 A$, the monthly number of outpatient visits in 2020 (255,932-425,234 per month) was significantly lower than that in the same period in 2019 (40,690-269,465 per month; $\mathrm{P}<0.05)$. Moreover, as shown in Figure 1B, the number and proportion of pediatric intestinal infections in $2020(1,602-10,818 ; 2.92-4.01 \%)$ were significantly lower than that in the same period in 2019 (18,065-28,014; 4.17-7.09\%; $\mathrm{P}<0.05)$.

We also compared the test results of adenovirus and rotavirus between 2019 and 2020. A total of 14,097 samples were tested in 2020, of which 223 were positive for adenovirus, and the positive rate was $1.58 \%$. The positive rate of adenovirus in 2020 was significantly lower than the level in $2019(2.69 \%, 815 / 30,285 ; \mathrm{P}<0.05)$. In 2020, the positive cases of rotavirus were 1,008 , and the positive rate was $7.15 \%$. The positive rate of rotavirus in 2020 is also significantly lower than the level in 2019 (14.41\%, $4,365 / 30,285 ; \mathrm{P}<0.05)$. The positive rate of rotavirus 

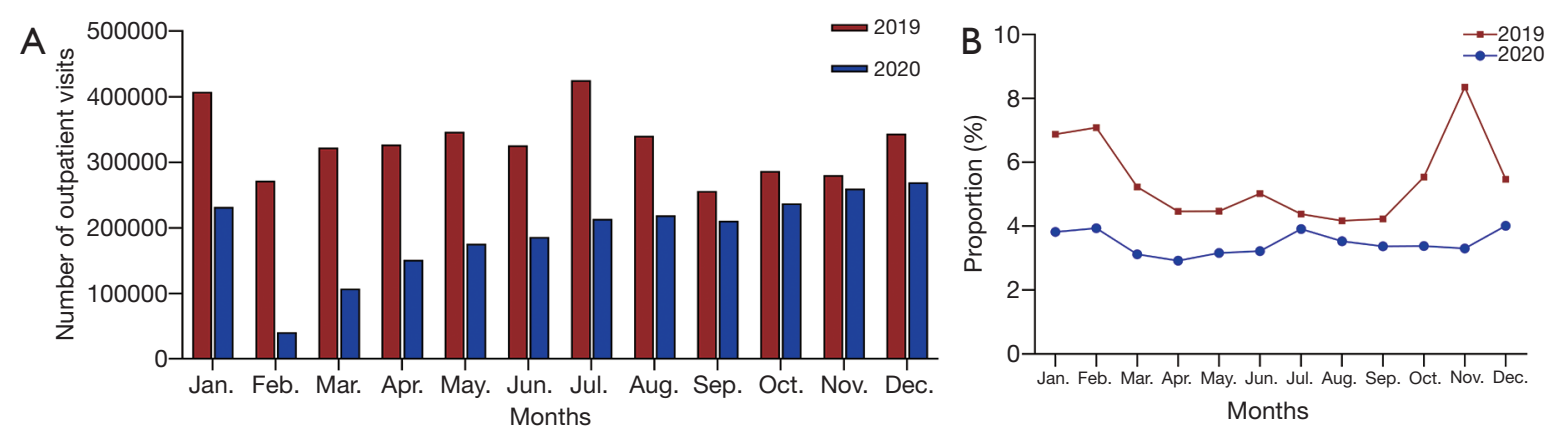

Figure 1 Distribution of patients with intestinal infection and outpatient visits from January-June in 2019 and 2020 . (A) Monthly number of outpatient visits; (B) proportion of patients with intestinal infection in outpatient visits.
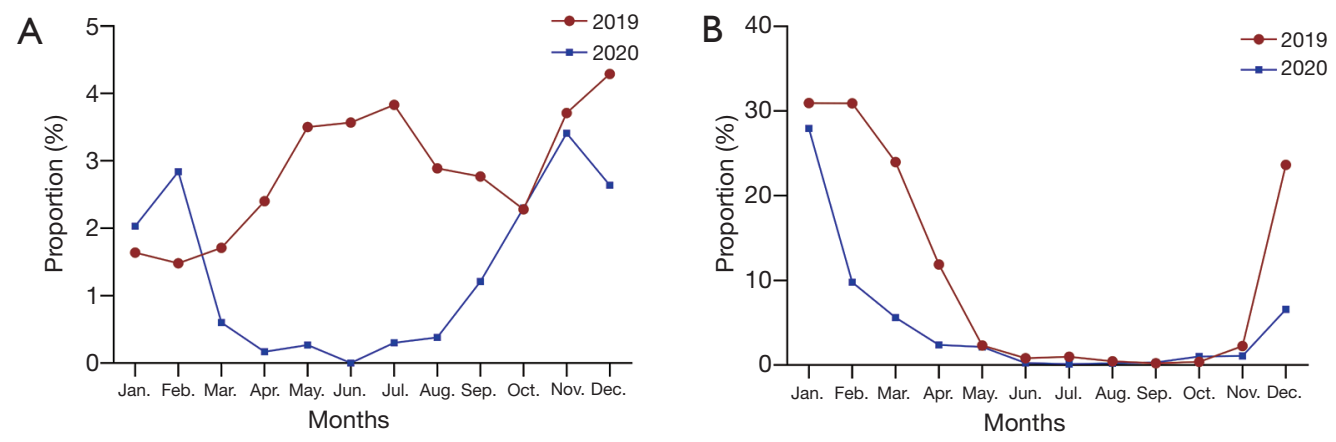

Figure 2 Distribution of positive rate for adenovirus and rotavirus from January to December in 2019 and 2020 . (A) Positive rate of adenovirus; (B) positive rate of rotavirus.

infection in boys was $11.71 \%(3,061 / 26,144)$, which was significantly lower than that of girls $(12.68 \%, 2,312 / 18,238$; $\mathrm{P}<0.05)$. Meanwhile, the positive rate of adenovirus infection in boys was $2.20 \%(574 / 26,144)$, which was significantly lower than that of girls $(2.54 \%, 464 / 18,328 ; \mathrm{P}<0.05)$.

The Figure 2 shows the distribution of completed tests for adenovirus and rotavirus from January to December in 2019 and 2020. As shown in Figure 2A, the positive rates of adenovirus from March to September and December of 2020 were $0.60 \%, 0.17 \%, 0.27 \%, 0,0.30 \%, 0.38 \%, 1.21 \%$, and $2.64 \%$ respectively, which were significantly lower than the levels of the same period in $2019(1.71 \%, 2.40 \%, 3.50 \%$, $3.57 \%, 3.83 \%, 2.89 \%, 2.77 \%, 4.29 \%$; $\mathrm{P}<0.05)$. As shown in Figure 2B, the positive rates of rotavirus from January to April and December of 2020 were 27.94\%, 9.79\%, 5.61\%, $2.38 \%$, and $6.59 \%$, respectively, which were significantly lower than the levels of rotavirus in the same period in 2019 (30.95\%, 30.90\%, 23.97\%, 11.89\%, 23.65\%; $\mathrm{P}<0.05$ ). From May to November in 2020 and 2019, the positive rate of rotavirus was all less than $5 \%$.
As shown in Figure 3, according to the age distribution, the positive rate of intestinal adenovirus infection in children aged 3-5 years is the highest during 2019-2020 (Figure 3A), while the positive rate of rotavirus infection in children aged 1-3 years is the highest (Figure 3B) during 2019-2020. In 2020 , the positive rate of adenovirus in children less than 6 months was $0.41 \%$, which was slightly higher than $0.34 \%$ in the same period in 2019 , and the positive rate of adenovirus in children of all age groups was lower than the level in the same period in 2019. Among them, the positive rates of adenovirus in the 6 months -1 year old, $1-3$ years old, and $3-5$ years old groups are $0.94 \%, 2.14 \%$, and $3.94 \%$, which were significantly lower than the adenovirus positive rates in the same period in 2019 (1.69\%, 2.82\%, 9.90\%; $\mathrm{P}<0.05)$. The positive rate of rotavirus in all age groups in 2020 is lower than the level of all age groups in 2019. Among them, the positive rate of rotavirus in the groups of $<6$ months, 6 months -1 year, 1-3 years, and 3-5 years old were $0.34 \%, 1.69 \%, 2.83 \%$, and $9.90 \%$, which significantly lower than the same age groups in 2019 (2.72\%, 9.75\%, 

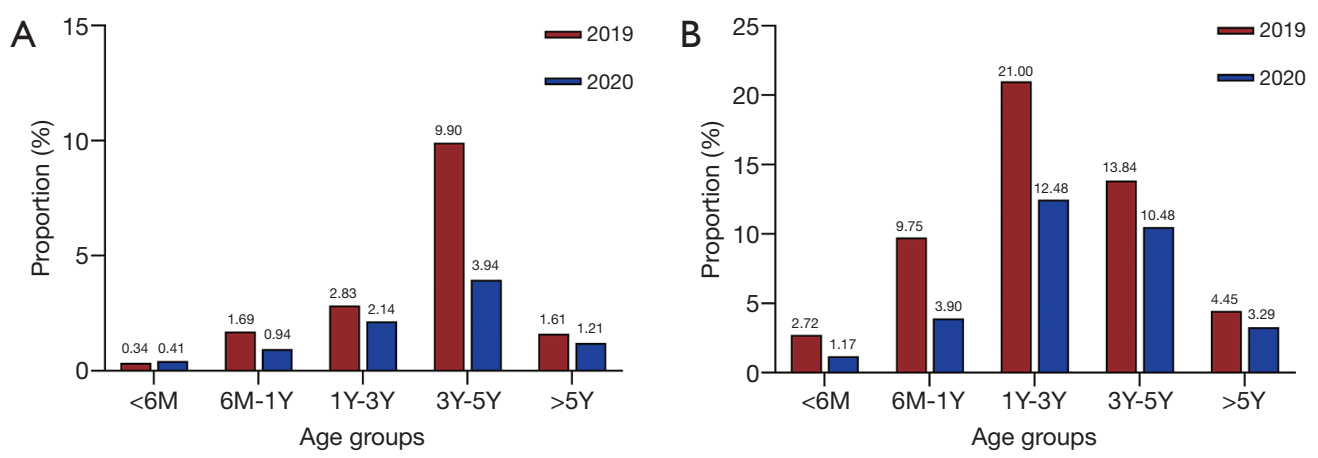

Figure 3 Distribution of positive rate for adenovirus and rotavirus in different age groups from January to December in 2019 and 2020 . (A) Positive rate of adenovirus infection; (B) positive rate of rotavirus infection.

$21.00 \%$, and $13.84 \%, \mathrm{P}<0.05)$.

\section{Discussion}

While posing a severe threat to global public health, the COVID-19 pneumonia epidemic has a substantial impact on spectrum of diseases worldwide. In this study, we found that pediatric outpatient visits from January to December of 2020 were significantly less than that in the same period of 2019. Furthermore, we found the numbers of outpatients and patients with intestinal infection were the lowest in February and increased slowly from March to June. It is probably due to the government's strict prevention and control policies against the serious novel coronavirus epidemic in February and March, hence decreasing the transmission of infectious virus. Moreover, the proportion of intestinal infection patients was significantly lower than that in the same period in 2019. This is because the spectrum of pediatric diseases had been changed during COVID-19 outbreak. The cases of injury, atopic dermatitis and emotional disorders increased significantly $(8,9)$. In our previous study, we found that respiratory tract infections in children significantly decreased during COVID-19 outbreak (10). Our results indicated that measures (restriction of travel, social distancing, home quarantine, etc.) during the COVID-19 outbreak decreased the incidence of intestinal infections.

Rotavirus is usually prevalent in winter (11). In January of 2020, there were a large number of positive cases and a high positive rate of rotavirus injection. However, both the positive number and rate of rotavirus infection dropped rapidly in February 2020. The positive rate of rotavirus in 2020 was constantly lower than that in 2019 from January to December. The monthly differences between 2019 and 2020 were most predominant in February, March and April. It indicated that the prevention and control measures of COVID-19 epidemic hindered the infection and transmission of rotavirus. Although the positive cases of adenovirus in 2020 was significantly lower than that in 2019 from March to December. In January and February of 2020 , there were higher positive rates of adenovirus than those in 2019. Under prevention and control measures, the positive rates of adenovirus in 2020 were lower than that in 2019 from March to September and December.

It was said that children under 2 years of age are the main infection groups of adenovirus (12), but our research results show that children aged 3-5 years old have the highest positive rate of adenovirus. This study found that children aged 1-3 years old are at a high risk of rotavirus infection. This result is consistent with previous studies (13). The positive rate of adenovirus and rotavirus by distribution of age in 2020 is exactly the same as in 2019, but the positive rate of adenovirus and rotavirus infection in 2020 is lower than the level of 2019, only the $<6$ months group of adenovirus except. This suggests that the COVID-19 pandemic has hardly affected the age distribution of intestinal adenovirus and rotavirus infections, but has reduced the positive rate of infection in all age groups as a whole. The positive rate of rotavirus and adenovirus in boy were all significantly lower than that of girl which mean the infectious rate may be related to gender.

For further studies, we will explore the epidemiological characteristics of children infected with adenovirus and rotavirus in Hangzhou in the post-epidemic, and provide post-epidemic data for the prevention and treatment of children with adenovirus and rotavirus in clinical. 


\section{Conclusions}

In summary, in the early phase of COVID-19 outbreak, the outpatients, the cases of intestinal infection, and positive cases of rotavirus or adenovirus slightly decreased under COVID-19 measures in Hangzhou. With the lift of control measures, the outpatients, the cases of intestinal infection were slowly increasing. The prevention and control of new coronavirus pandemic can also limit the infection and transmission of rotavirus and adenovirus.

\section{Acknowledgments}

Funding: This study was funded by the science and technology projects in Zhejiang Province (LGC21H200004 and 2019C03037) and the Medical Scientific Projects from Health Department of Zhejiang Province (2018KY455).

\section{Footnote}

Reporting Checklist: The authors have completed the MDAR reporting checklist. Available at https://dx.doi. org/10.21037/tp-21-150

Data Sharing Statement: Available at https://dx.doi. org/10.21037/tp-21-150

Peer Review File: Available at https://dx.doi.org/10.21037/ tp-21-150

Conflicts of Interest: All authors have completed the ICMJE uniform disclosure form (available at https://dx.doi. org/10.21037/tp-21-150). The authors have no conflicts of interest to declare.

Ethical Statement: The authors are accountable for all aspects of the work in ensuring that questions related to the accuracy or integrity of any part of the work are appropriately investigated and resolved. The study was conducted in accordance with the Declaration of Helsinki (as revised in 2013). The study was approved by the ethics committee of the Children's Hospital of Zhejiang University School of Medicine (2020-IRB-016) and informed consent was taken from all the patients.

Open Access Statement: This is an Open Access article distributed in accordance with the Creative Commons Attribution-NonCommercial-NoDerivs 4.0 International
License (CC BY-NC-ND 4.0), which permits the noncommercial replication and distribution of the article with the strict proviso that no changes or edits are made and the original work is properly cited (including links to both the formal publication through the relevant DOI and the license). See: https://creativecommons.org/licenses/by-nc-nd/4.0/.

\section{References}

1. Chen J, Wan CM, Gong ST, et al. Chinese clinical practice guidelines for acute infectious diarrhea in children. World J Pediatr 2018;14:429-36.

2. Li W, Xiang W, Li C, et al. Molecular epidemiology of rotavirus $\mathrm{A}$ and adenovirus among children with acute diarrhea in Hangzhou, China. Gut Pathog 2020;12:19.

3. Dian Z, Fan M, Wang B, et al. The prevalence and genotype distribution of rotavirus A infection among children with acute gastroenteritis in Kunming, China. Arch Virol 2017;162:281-5.

4. Li L, Phan TG, Nguyen TA, et al. Molecular epidemiology of adenovirus infection among pediatric population with diarrhea in Asia. Microbiol Immunol 2005;49:121-8.

5. Pan A, Liu L, Wang C, et al. Association of Public Health Interventions With the Epidemiology of the COVID-19 Outbreak in Wuhan, China. JAMA 2020;323:1915-23.

6. Li Z, Chen Q, Feng L, et al. Active case finding with case management: the key to tackling the COVID-19 pandemic. Lancet 2020;396:63-70.

7. Xiang $\mathrm{W}$, Peng $\mathrm{Z}$, Xu J, et al. Evaluation of a commercial latex agglutination test for detecting rotavirus $\mathrm{A}$ and human adenovirus in children's stool specimens. J Clin Lab Anal 2020;34:e23208.

8. Li H, Yu G, Duan H, et al. Changes in Children's Healthcare Visits During Coronavirus Disease-2019 Pandemic in Hangzhou, China. J Pediatr 2020;224:146-9.

9. Jiao WY, Wang LN, Liu J, et al. Behavioral and Emotional Disorders in Children during the COVID-19 Epidemic. J Pediatr 2020;221:264-266.e1.

10. Zhu Y, Li W, Yang B, et al. Epidemiological and virological characteristics of respiratory tract infections in children during COVID-19 outbreak. BMC Pediatr 2021;21:195.

11. Chen YH, Chen F, Zhou T, et al. Prevalence and clinical profile of rotavirus A infection among diarrhoeal children and phylogenetic analysis with vaccine strains in Chengdu, West China, 2009-2014. Trop Med Int Health 2018;23:704-13.

12. Esposito S, Zampiero A, Bianchini S, et al. Epidemiology 
and Clinical Characteristics of Respiratory Infections Due to Adenovirus in Children Living in Milan, Italy, during 2013 and 2014. PLoS One 2016;11:e0152375.

Cite this article as: Li W, Zhu Y, Lou J, Chen J, Xie X, Mao J. Rotavirus and adenovirus infections in children during COVID-19 outbreak in Hangzhou, China. Transl Pediatr 2021;10(9):2281-2286. doi: 10.21037/tp-21-150
13. Lou JT, Xu XJ, Wu YD, et al. Epidemiology and burden of rotavirus infection among children in Hangzhou, China. J Clin Virol 2011;50:84-7. 